\title{
Analyticity Properties of the Feigenbaum Function
}

\author{
H. Epstein ${ }^{1}$ and J. Lascoux ${ }^{2}$ \\ 1 Institut des Hautes Etudes Scientifiques, F-91440 Bures-sur-Yvette, France \\ 2 Centre de Physique Théorique, Ecole Polytechnique, F-91120 Palaiseau, France
}

\begin{abstract}
Analyticity properties of the Feigenbaum function [a solution of $g(x)=-\lambda^{-1} g(g(\lambda x))$ with $\left.g(0)=1, g^{\prime}(0)=0, g^{\prime \prime}(0)<0\right]$ are investigated by studying its inverse function which turns out to be Herglotz or anti-Herglotz on all its sheets. It is found that $g$ is analytic and uniform in a domain with a natural boundary.
\end{abstract}

\section{Introduction}

In the theory of successive period doublings of one-parameter families of smooth mappings of the interval $[-1,1]$, an important role is played by one particular such function, here denoted $g$, which is a solution of a certain functional equation [see Eq. (1) below]. This theory is expounded at length in references $[5,6,2-4,8]$ and will not be recalled here. The purpose of this note is to indicate a few analyticity properties of this function, which might, in the future, throw some light on the still somewhat mysterious aspects of this theory.

Proofs of the existence of $g$ have been provided successively by Lanford [7, 9], Campanino et al. [1], and again by Lanford [10]. None of them is truly satisfactory (see comments in [8]).

\subsection{Notations}

We denote $\Pi_{+}=-\Pi_{-}=\{\zeta \in \mathbb{C}: \operatorname{Im} \zeta>0\}$ the open upper half plane. $\bar{\zeta}$ will always denote the complex conjugate of $\zeta \in \mathbb{C}$, and, to avoid confusions, the closure of a set $E$ will be denoted $E^{c}$. A holomorphic function $\varphi$ of a complex variable is called "selfconjugate" if $\varphi(\zeta)=\bar{\varphi}(\bar{\zeta})$. 


\section{Recapitulation of Known Properties}

a. The method described in [1] proves that there exists a solution $g$ of the Cvitanović-Feigenbaum-Coullet-Tresser functional equation:

$$
\left.\begin{array}{l}
g(x)=-\frac{1}{\lambda} g(g(\lambda x)), \quad-1 \leqq x \leqq 1, \\
g(0)=1,
\end{array}\right\} .
$$

with the following properties:

1) $g$ is analytic in a complex neighborhood of $[-1,1]$.

2) $g$ is even and concave: $g^{\prime \prime}(x)<0$ for all $x \in[-1,1]$ and in particular $g^{\prime \prime}(0)=-2 \alpha$, with $1.429<\alpha<1.615$.

3) $\lambda=-g(1)=-g^{\prime}(1)^{-1}>0$; in fact: $0.152<\lambda^{2}<0.165$.

From these properties and the functional equation(1), it follows immediately that $g$ extends to a real analytic function on $\mathbb{R}$ and also on $i \mathbb{R}$; the function $f$, initially defined on $[0,1]$ by $f(t)=g(\sqrt{t})$, extends to a real analytic function over $\mathbb{R}$. Moreover, slightly more detailed information obtained in the course of the proof in [1] easily shows that the graphs of $f$ and $g$ have the appearance given in Figs. 1 and 2. By construction, the graph of $g$ restricted to $\left[-\lambda^{-n}, \lambda^{-n}\right], n \geqq 1$, is obtained by a dilation $(-\lambda)^{-n}$ from the graph of the $\left(2^{n}\right)^{\text {th }}$ iterate of $g \mid[-1,1]$. Hence $|x| \leqq \lambda^{-n} \Rightarrow|g(x)| \leqq \lambda^{-n}$, and $|x| \geqq 1 \Rightarrow|g(x)|<\lambda^{-1}|x|$. Another consequence is that the succession of critical points of $g$ on $\mathbb{R}$ is dictated by the known kneading sequence of $g \mid[-1,1]$ and that these critical points are simple and form an infinite sequence ${ }^{1}$. Let $J_{k},(k=1,2,3, \ldots)$ be the $k^{\text {th }}$ intercritical interval on the positive real axis, i.e. $J_{1}=\left(0, x_{0} / \lambda\right)$, $J_{2}=\left(x_{0} / \lambda, x_{1}\right), J_{3}=\left(x_{1}, x_{0} / \lambda^{2}\right)$, etc. $\ldots$. For $-k=1,2,3, \ldots$, we define $J_{k}=-J_{-k}$. Let $g_{k}=g \mid J_{k}$. For each $k$ with $|k|>1$, there exist $j$ and $\ell$ with $|j|<|k|,|\ell|<|k|$, such that, $\forall x \in J_{k}$,

$$
g_{k}(x)=-\frac{1}{\lambda} g_{j}\left(g_{t}(\lambda x)\right)
$$

This is easily seen by induction on $k$. The ends of $J_{k}$ are reached when either $g_{j}$ or $g_{\ell}$ acquires a critical point. (The argument showing that these 2 events cannot be simultaneous, (due to Lanford) consists in noting that this would imply a superstable periodic orbit for $g \mid[-1,1]$.) The following "multiplication table" (Table 1) shows values of $j$ and $\ell$ for the first few values of $k>0$. It is also obtainable from the kneading sequence.

The functions $g$ and $f$ have negative Schwarzian derivative, i.e.

$$
S g(x) \equiv g^{\prime \prime \prime}(x) g^{\prime}(x)^{-1}-\frac{3}{2}\left[g^{\prime \prime}(x) g^{\prime}(x)^{-1}\right]^{2} \leqq 0, \quad x \in \mathbb{R}
$$

(and similarly for $f$ ). For $0 \leqq x \leqq 1,0 \leqq t \leqq 1$, we have

$$
\begin{array}{cc}
g^{\prime}(x) \leqq 0, & g^{\prime \prime}(x)<0, \quad g^{\prime \prime \prime}(x) \geqq 0, \\
f^{\prime}(t)<0, & f^{\prime \prime}(t)>0, \quad f^{\prime \prime \prime}(t)>0 .
\end{array}
$$

1 We learned this fact from O.E. Lanford 
Table 1

\begin{tabular}{lrrrrrrrrrrrrr}
\hline$k$ & 2 & 3 & 4 & 5 & 6 & 7 & 8 & 9 & 10 & 11 & 12 & 13 & 14 \\
\hline$j$ & -1 & -2 & -2 & -1 & 1 & 2 & 3 & 4 & 5 & 5 & 4 & 3 & 3 \\
\hline$l$ & 1 & 1 & 2 & 2 & 2 & 2 & 2 & 2 & 2 & 3 & 3 & 3 & 4 \\
\hline
\end{tabular}

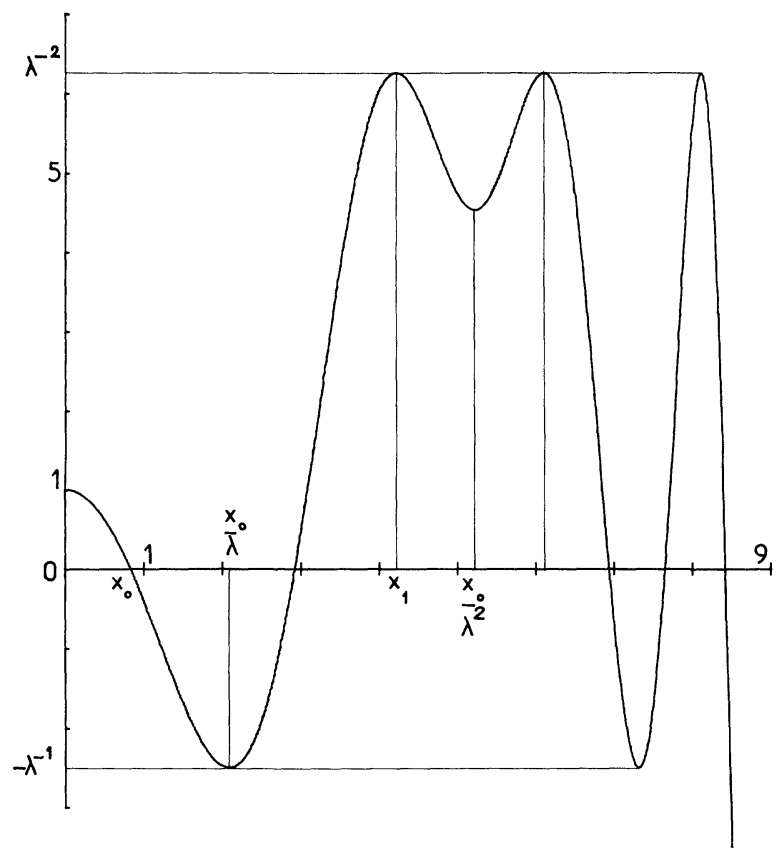

Fig. 1. Graph of the function $g$

In the interval $[0,1]$ there is one solution $x_{0}$ of $x_{0}=g\left(\lambda x_{0}\right)$; Eq. (1) then shows that $g\left(x_{0}\right)=0$ : this is the unique zero of $g$ in this interval, and it satisfies: $g^{\prime}\left(\lambda x_{0}\right)=-1$, $f\left(x_{0}^{2}\right)=0$. The convexity of $f$ and the concavity of $g$, together with: $g(1)=-\lambda$, $g^{\prime}(1)=-\lambda^{-1}, f(1)=-\lambda, f^{\prime}(1)=-1 / 2 \lambda$ shows that $\left(1-2 \lambda^{2}\right)<x_{0}^{2}<\left(1-\lambda^{2}\right)^{2}$.

b. Considerably more detail is provided by Lanford's first proof $[7,9]$ which has the advantage of yielding (in particular) the Taylor series of $f$ at 0 with any desired degree of accuracy. These numbers, kindly communicated to us by their author, are the basis of the various plots shown in this paper. Furthermore, they have been used by Lanford [9] to prove the existence of a singularity of $g$ in the complex plane (at the point $c$ later to be reobtained in this paper). Briefly and incompletely the argument is as follows : one proves, (using the Taylor series and its known degree of accuracy) that $z \rightarrow g(\lambda z)$ has a periodic point $c$ within its domain of analyticity: $g(\lambda c)=\bar{c}, g(\lambda \bar{c})=c$, $c \neq \bar{c}$. If $g$ could be continued to $c$, it would satisfy $g(c)=-\lambda^{-1} g(\bar{c})=-\lambda^{-1} \bar{g}(c)$, hence $|g(c)|=0$; thus for a certain $a \neq 0$ and $n \geqq 1$,

$$
g(z)=(z-c)^{n}[a+(z-c) r(z)]
$$




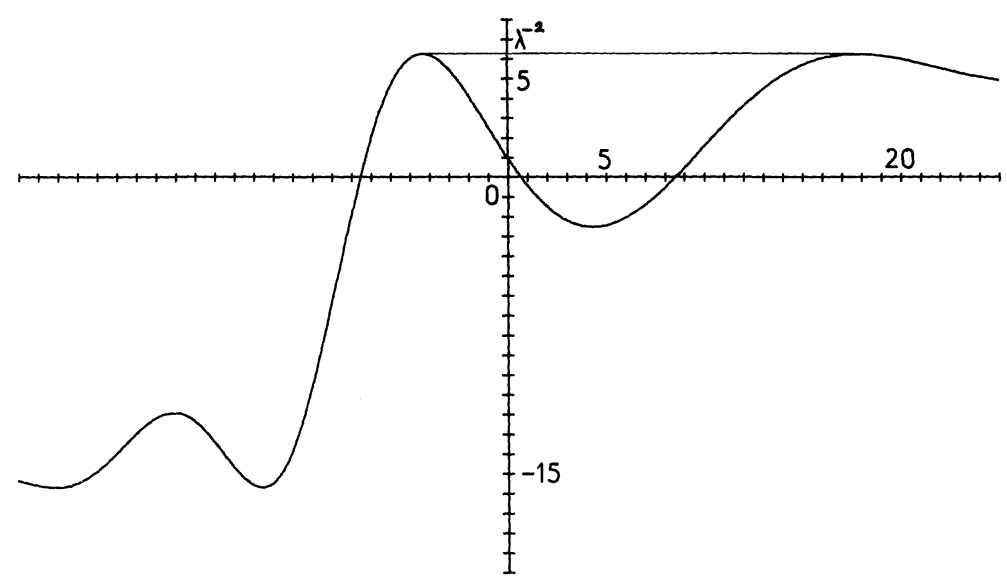

Fig. 2. Graph of the function $f$

around $c$. The functional equation then gives

$$
\begin{aligned}
g(z) & =-\lambda^{-1} g\left(\bar{c}+g^{\prime}(\lambda c) \lambda(z-c)+\ldots\right) \\
& =-\lambda^{-1}\left[g^{\prime}(\lambda c) \lambda(z-c)\right]^{n}[\bar{a}+(z-c) s(z)]
\end{aligned}
$$

and therefore $\lambda^{-1}\left|\lambda g^{\prime}(\lambda c)\right|^{n}=1$. This is not the case, and a more detailed investigation shows that, in fact, there is a sequence $\left\{z_{n}\right\}$ such that $z_{n} \rightarrow c$ and $\left|g\left(z_{n}\right)\right| \rightarrow \infty$.

\section{The Inverse Function of $g$}

For each $k \in \mathbb{Z}$ we denote $u_{k}$ the inverse function of the restriction $g_{k}$ of $g$ to $J_{k}$. Since $g_{k}(x)=g_{-k}(-x)$, we have $u_{k}=-u_{-k}$. From (2) it follows that, with the same $k, j, \ell$

$$
u_{k}(\zeta)=\frac{1}{\lambda} u_{\ell}\left(u_{j}(-\lambda \zeta)\right)
$$

The function $u_{1}$, also denoted $u$, satisfies

$$
u(\zeta)=\frac{1}{\lambda} u(u(-\lambda \zeta)), \quad-\frac{1}{\lambda}<\zeta<1
$$

It is clear that each $u_{k}$ is analytic in a complex neighborhood of $g_{k}\left(J_{k}\right)$, and, by general theorems, has a square-root-type branch point at each end of that interval. The function $f$ also has an infinity of intervals of monotonicity. Using the estimates of [1] one can prove that, for a certain $t_{1}>0, t_{1}<6, f$ is monotonic decreasing with $f^{\prime}(t)<0$ in $\left(-t_{1}, x_{0}^{2} \lambda^{-2}\right)$, taking the values $f\left(-t_{1}\right)=\lambda^{-2}, f\left(x_{0}^{2}\right)=0, f\left(x_{0}^{2} \lambda^{-2}\right)=-\lambda^{-1}, f^{\prime}\left(-t_{1}\right)$ $=f^{\prime}\left(x_{0}^{2} \lambda^{-2}\right)=0$. The inverse function $U$ of the restriction of $f$ to that interval, [which satisfies $U(\zeta)=u(\zeta)^{2}$ for all $\left.\zeta \in\left(-\lambda^{-1}, 1\right)\right]$ is defined, real and analytic in $\left(-\lambda^{-1}, \lambda^{-2}\right)$, and negative in $\left(1, \lambda^{-2}\right)$. This displays the trivial nature of the singularity of $u$ at 1 .

We now study the analytic continuation of $u$. We recall the following classical facts $[11,12]$. 
Lemma 1. Let $\left\{\phi_{n}\right\}$ be a sequence of functions holomorphic in $\Delta=\Pi_{+} \cup \Pi_{-} \cup(a, b)$, (where $(a, b)$ is a non-empty open real interval) and having the properties: $\phi_{n}\left(\Pi_{+}\right) \subset \Pi_{+}, \phi_{n}\left(\Pi_{-}\right) \subset \Pi_{-}, \phi_{n}((a, b)) \subset(c, d) \subset \mathbb{R}$, where $-\infty<c<d \leqq \infty$. Suppose that the sequence $\left\{\phi_{n}\right\}$ converges uniformly over a compact subinterval $K \subset(a, b)$. Then $\left\{\phi_{n}\right\}$ converges, uniformly in any compact subset of $\Delta$, to a function $\phi$, holomorphic in $\Delta$, with $\phi\left(\Pi_{+}\right) \subset \Pi_{+}, \phi\left(\Pi_{-}\right) \subset \Pi_{-}, \phi((a, b)) \subset(c, d)$.

If, moreover, $\phi_{n}$ is injective in $\Delta$ for all $n$, then either $\phi$ is a constant or it is injective.

The first part immediately reduces to Vitali's theorem after $\Pi_{+} \cup \Pi_{-} \cup(c, d)$ has been mapped onto the unit disk by a conformal map $\tau$ and the sequence $\left\{\phi_{n}\right\}$ replaced by the bounded sequence $\left\{\tau \circ \phi_{n}\right\}$ (cf. [12]). The second part is similar to Hurwitz's theorem and can be found, e.g., in Rudin's textbook [11] as the last step in the proof of Riemann's theorem on conformal mapping.

We now prove:

Lemma 2. uextends to a function holomorphic in $\Pi_{+} \cup \Pi_{-} \cup\left(-\lambda^{-1}, 1\right)$, (again denoted $u$ or $u_{1}$ ) which is injective there and verifies

(i) $u(\zeta)=\bar{u}(\bar{\zeta})$,

(ii) $u(\zeta)=\frac{1}{\lambda} u(u(-\lambda \zeta))$,

(iii) $\operatorname{Im} \zeta>0 \Rightarrow(\operatorname{Im} u(\zeta)<0$ and $\operatorname{Re} u(\zeta)>0)$.

Analytic functions mapping $\Pi_{+}$into $\Pi_{+}$and $\Pi_{-}$into $\Pi_{-}$will be called Herglotz functions; if $-h$ is a Herglotz function, then $h$ is called an anti-Herglotz function.

Proof of Lemma 2. In [1], $g$ is obtained as the fixed point of a contractive map defined as follows. Starting with a real function $\varphi_{1}$ on $[0,1]$ with $\varphi_{1}(0)=1, \varphi_{1}^{\prime}(1)=-\lambda^{-1}$, one defines

$$
F(x)=\frac{1}{\lambda}\left[\varphi_{1}(1-x)-\varphi_{1}(1)\right]
$$

and constructs (by iteration) a function $\Psi$ such that

$$
\Psi(t)=F\left(\Psi\left(\lambda^{2} t\right)\right), \quad \Psi(0)=0, \quad \Psi^{\prime}(0)=1 .
$$

Then, defining $\alpha$ as the smallest solution of

$$
2 \alpha \lambda \Psi^{\prime}(\alpha)=1, \quad \alpha>0,
$$

one defines

$$
\begin{gathered}
\varphi_{2}(x)=1-\Psi\left(\alpha x^{2}\right), \\
G(x)=\frac{1}{\lambda}\left(\varphi_{2}(1-x)-\varphi_{2}(1)\right)=\frac{1}{\lambda}\left[\Psi(\alpha)-\Psi\left(\alpha(1-x)^{2}\right)\right] .
\end{gathered}
$$

The mapping in question is $\varphi_{1} \rightarrow \varphi_{2}$ or, equivalently the mapping $T_{\lambda}$ given by $T_{\lambda} F=G$. It depends on $\lambda$ as a parameter, and, for $0.152 \leqq \lambda^{2} \leqq 0.165$, is defined and contractive on a set of functions $F$ which can be described as follows:

$F$ must be $\mathscr{C}^{3}$ on $[0,1], F(0)=0, F^{\prime}(0)=\lambda^{-2}$ and for all $x \in(0,1)$,

$$
F^{\prime}(x) \geqq 0, \quad F^{\prime \prime}(x) \leqq 0, \quad F^{\prime \prime \prime}(x) \leqq 0, \quad-F^{\prime \prime}(x) / F^{\prime}(x) \leqq(1-x)^{-1} .
$$


For $0 \leqq x \leqq A$,

$$
\begin{aligned}
\frac{1}{1-x}-\ell_{1}(1-x)-\ell_{3}(1-x)^{3} & \leqq-\frac{F^{\prime \prime}(x)}{F^{\prime}(x)} \leqq \frac{1}{1-x}-c_{1}(1-x)-c_{3}(1-x)^{3} ; \\
\frac{d}{d x}\left(-\frac{F^{\prime \prime}(x)}{F^{\prime}(x)}\right) & \leqq L .
\end{aligned}
$$

Here $A, c_{1}, c_{3}, \ell_{1}, \ell_{3}$, and $L$ are piecewise constant positive functions of $\lambda$. [In the end $\lambda$ is chosen so that the fixed point of $T_{\lambda}$ yields a solution of (1).] If $F$ belongs to this subset then so does $G=T_{\lambda} F$, and $G$ satisfies the condition (6) for all $x \in[0,1]$. If $F$ is chosen in a certain class of functions holomorphic near $[0,1], G$ also belongs to it; $\Psi$ is then also analytic. $\Psi$ is obtained as the limit of $\Psi_{m}$ when $m \rightarrow \infty$, where $\Psi_{0}(t)=t$ and $\Psi_{m+1}(t)$ $=F\left(\Psi_{m}\left(\lambda^{2} t\right)\right), t \in\left[0, \lambda^{-2}\right]$.

It is clear that $F, \Psi_{m}$, and $\Psi$ all have $\mathscr{C}^{3}$ (or, in fact, if $F$ is analytic, analytic) inverse functions on $(0,1),\left(0, \lambda^{-2}\right)$ respectively, and that $\Psi_{m}^{-1}$ converges to $\Psi^{-1}$. These functions satisfy

$$
\Psi_{m+1}^{-1}(\zeta)=\lambda^{-2} \Psi_{m}^{-1}\left(F^{-1}(\zeta)\right)
$$

This holds for all $m \geqq 0$ and all $\zeta \in F([0,1])$. It follows from (5) that $F(x) \geqq \lambda^{-2} x(1-x / 2)$ for $x \in[0,1]$, and (inductively on $m$, by estimates similar to those in $[1$, Sect. 5])

$$
1-\lambda^{2} t /\left(1-\lambda^{2}\right) \leqq \Psi_{m}^{\prime}(t) \leqq 1, \quad t\left[1-\lambda^{2} t / 2\left(1-\lambda^{2}\right)\right] \leqq \Psi_{m}(t) \leqq t
$$

for all $t \in\left[0, \lambda^{-2}\right]$. In particular $F(1) \geqq \lambda^{-2} / 3>2$ and $\Psi_{m}\left(\lambda^{-2}\right) \geqq \lambda^{-2}\left[1-1 / 2\left(1-\lambda^{2}\right)\right]>2$. Since $F$ and $\Psi_{m}$ are increasing, it follows that $F^{-1}$ is defined on $\left[0, \lambda^{-2} / 3\right], \Psi_{m}^{-1}$ is defined on $[0,2]$ and $F^{-1}(\zeta) \leqq 1$ for $0 \leqq \zeta \leqq \lambda^{-2} / 3$. Assume now that $F^{-1}$ extends to a function holomorphicin $\Pi_{+} \cup \Pi_{-} \cup\left(0, \lambda^{-2} / 3\right)$ and maps $\Pi_{+}$injectively into $\{w \in \mathbb{C}: \operatorname{Im} w>0, \operatorname{Re} w<1\}$. Assume that, for some $m, \Psi_{m}^{-1}$ extends to a function holomorphic in $\Pi_{+} \cup \Pi_{-} \cup(0,2)$ and maps $\Pi_{+}$injectively into itself; (this is certainly true for $m=0$. Since $F^{-1}$ and $\Psi_{m}^{-1}$ are self-conjugate, there are symmetric statements about their behavior in $\left.\Pi_{-}\right)$. Then $\Psi_{m+1}^{-1}$ has the same property as $\Psi_{m}^{-1}$ and, by Lemma 1 , so does $\Psi^{-1}$. From the equation

$$
G^{-1}(\zeta)=1-\left[\alpha^{-1} \Psi^{-1}(\Psi(\alpha)-\lambda \zeta)\right]^{1 / 2},
$$

which holds for $0 \leqq \zeta \leqq \lambda^{-1} \Psi(\alpha)$, with the square root defined as positive, it follows that $G^{-1}$ extends to a function holomorphic in $\Pi_{+} \cup \Pi_{-} \cup\left(0, \lambda^{-2} / 3\right)$ : for $0 \leqq \zeta$ $\leqq \lambda^{-2} / 3$ we find $\lambda \zeta<1<\Psi(\alpha)<\alpha<2$. For $\zeta \in \Pi_{+}$, we have $\alpha^{-1} \Psi^{-1}(\Psi(\alpha)-\lambda \zeta) \in \Pi_{-}$, and therefore

$$
G^{-1}(\zeta) \in\{w \in \mathbb{C}: \operatorname{Im} w>0, \operatorname{Re} w<1\} .
$$

Thus $G^{-1}$ is analytic in the same domain as $F^{-1}$. Moreover $G^{-1}$ is injective in $\Pi_{+}$ since $G^{-1}\left(\zeta_{1}\right)=G^{-1}\left(\zeta_{2}\right), \zeta_{1,2} \in \Pi_{+}$, implies

$$
\Psi^{-1}\left(\Psi(\alpha)-\lambda \zeta_{1}\right)=\Psi^{-1}\left(\Psi(\alpha)-\lambda \zeta_{2}\right) \Rightarrow \zeta_{1}=\zeta_{2}
$$

Now iterating the mapping $T_{\lambda}: F \rightarrow G$ and applying Lemma 1 completes the proof of Lemma 2, provided an initial $F$ with the required properties can be found. Such examples are given in the appendix. 
From now on, $u=u_{1}$ will denote the function analytic and injective in $\Pi_{+} \cup \Pi_{-} \cup\left(-\lambda^{-1}, 1\right)$ whose existence is asserted in Lemma 2 , and $U$ will denote the function, analytic and injective in $\Pi_{+} \cup \Pi_{-} \cup\left(-\lambda^{-1}, \lambda^{-2}\right)$ which satisfies $U(\zeta)=u(\zeta)^{2}$ in $\left(-\lambda^{-1}, 1\right)$. Both $u$ and $U$ are anti-Herglotz functions.

\section{Sheet Structure of the Analytic Continuation of $u$}

\subsection{The Functions $u_{k}, k \in \mathbb{Z}$}

The branch of the analytic continuations of $u$ that is easiest to study is $u_{-1}$ since it is simply given by $u_{-1}(\zeta)=-u(\zeta)$. It is therefore a Herglotz function with

$$
u_{-1}\left(\Pi_{+}\right) \subset\{w \in \mathbb{C}: \operatorname{Im} w>0, \operatorname{Re} w<0\} .
$$

It communicates with $u$ across the segment $I_{0}=\left(1, \lambda^{-2}\right)$ of $\mathbb{R}$, i.e. when $\zeta$ crosses $I_{0}$ from $\Pi_{+}$to $\Pi_{-}, u(\zeta)$ gets analytically continued by $u_{-1}(\zeta)$ while the value $u(\zeta)$ crosses the imaginary axis from the right to the left half-plane. The branch point at 1 is of the square-root type and $u(\zeta \pm i 0)$ are continuous there.

According to Table 1,

$$
u_{2}(\zeta)=\frac{1}{\lambda} u_{1}\left(-u_{1}(-\lambda \zeta)\right)
$$

Hence $u_{2}$ is a Herglotz function (injective in $\Pi_{ \pm}$) which communicates with $u_{1}$ across the cut between $-\lambda^{-1}$ and $-\lambda^{-3}$. The nature of the branch point at $-\lambda^{-1}$ is again trivial: as $\zeta$ approaches $-\lambda^{-1}, u_{2}(\zeta)$, as given by (11), has a singularity only because $-\lambda \zeta$ approaches 1 , so that $u_{1}(-\lambda \zeta)$ behaves like $\sqrt{1+\zeta \lambda}$, while the outer $u_{1}$, being holomorphic near 0 , just gives a holomorphic image of this behavior. The same occurs for all the $u_{k}, k \in \mathbb{Z}$. Each $u_{k}$, given by

$$
u_{k}(\zeta)=\lambda^{-1} u_{\ell}\left(u_{j}(-\lambda \zeta)\right)
$$

is a Herglotz or anti-Herglotz function. As $\zeta$ approaches one of the ends of its real interval of analyticity $u_{k}\left(J_{k}\right)$, only one of the functions $u_{\ell}$ or $u_{j}$ has a singularity (by Lanford's argument) which, by induction, is a trivial square root branch point. Figure 3 describes the situation by analogy with the cosine function.

\subsection{Boundary of $u\left(\Pi_{-}\right)$}

We now study the boundary values of $u$ along the real axis. Denote

$$
I_{0}=\left(1, \lambda^{-2}\right), \quad I_{n}=-\lambda^{-1} I_{n-1}=(-1)^{n}\left(\lambda^{-n}, \lambda^{-n-2}\right), \quad(n=1,2,3, \ldots) .
$$

As $\zeta$ follows $I_{0}-i 0, u(\zeta)$ follows the segment $\tau_{0}=i\left(0, \sqrt{t_{1}}\right)$ of the imaginary axis. If $\zeta$ crosses $I_{0}$ into $\Pi_{+}, u(\zeta)$ gets continued by $u_{-1}(\zeta)=-u(\zeta)$ and the value of $u(\zeta)$ crosses $\tau_{0}$ into $-u\left(\Pi_{+}\right)$. At $\zeta=1$, as noted, $u(\zeta-i 0)$ is continuous. Suppose now $\zeta$ follows $I_{1}-i 0$. Then $-\lambda \zeta$ follows $I_{0}+i 0, u(-\lambda \zeta)$ follows $\bar{\tau}_{0}$ which is inside the domain of analyticity of $u$. Hence

$$
u(\zeta)=\lambda^{-1} u(u(-\lambda \zeta))
$$




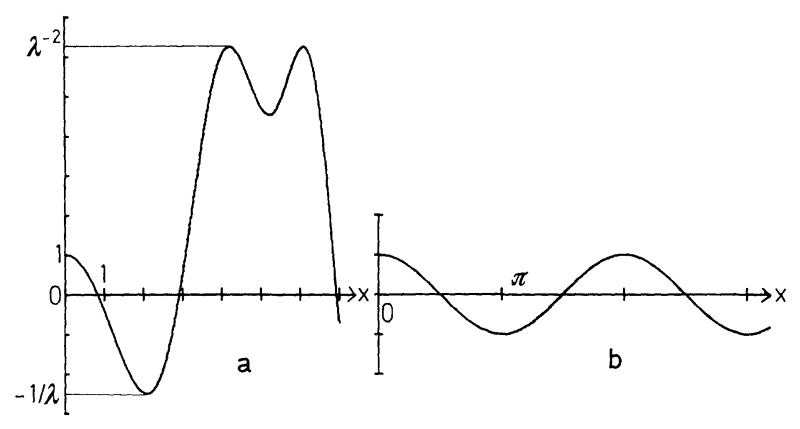

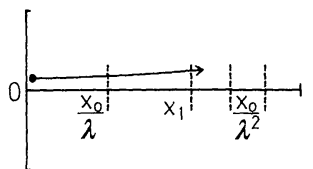

C

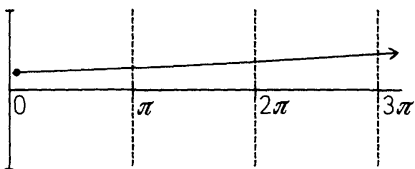

d

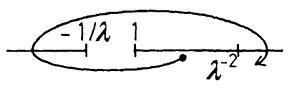

e

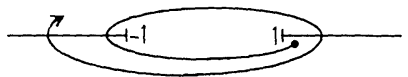

f

Fig. 3a-f. The analogy between $g$ and cos locally describes the sheets whose images border the real axis:a: graph of $x \rightarrow g(x) ; \mathbf{b}:$ graph of $x \rightarrow \cos x ; \mathbf{c}$ and $\mathbf{d}:$ positions of images of sheets of inverse functions of $g$ and cos near $\mathbb{R}$. As $x$ becomes complex and follows the contours indicated in $\mathbf{c}$ and $\mathbf{d}$, respectively, $g(x)$ and $\cos (x)$ follow the contour indicated in $\mathbf{e}$ and $\mathbf{f}$, respectively

follows $\frac{1}{\lambda} u\left(\bar{\tau}_{0}\right) \equiv \tau_{1}$. This is a smooth curve in $\Pi_{+}$which is part of $\frac{1}{\lambda} u(i \mathbb{R})$, and starts at $\frac{1}{\lambda} u(0)=x_{0} / \lambda$ perpendicularly to the real axis. If $\zeta$ crosses $I_{1}$ into $\Pi_{+}$then $-\lambda \zeta$ crosses $I_{0}$ into $\Pi_{-}$, where $u(-\lambda \zeta)$ gets continued by $-u(-\lambda \zeta)$ while its value remains inside the domain of analyticity of $\frac{1}{\lambda} u$; thus $u(\zeta)$ gets continued by

$$
v_{1}(\zeta) \equiv \lambda^{-1} u(-u(-\lambda \zeta))
$$

By induction it is immediately seen that: as $\zeta$ follows $I_{n}-i 0,(n \geqq 1), u(\zeta)$ follows a smooth piece of curve $\tau_{n} \subset \Pi_{+}$; at the same time $-\lambda \zeta$ follows $I_{n-1}+i 0, u(-\lambda \zeta)$ follows $\bar{\tau}_{n-1} \subset \Pi_{-}$and $\tau_{n}=\frac{1}{\lambda} u\left(\bar{\tau}_{n-1}\right)$. If $\zeta$ crosses into $\Pi_{+}, u(\zeta)$ gets continued by

$$
v_{n}(\zeta)=\frac{1}{\lambda} u\left(v_{n-1}(-\lambda \zeta)\right)=\ldots=\left(\frac{1}{\lambda} u\right)^{n}\left(-u\left((-\lambda)^{n} \zeta\right)\right) .
$$




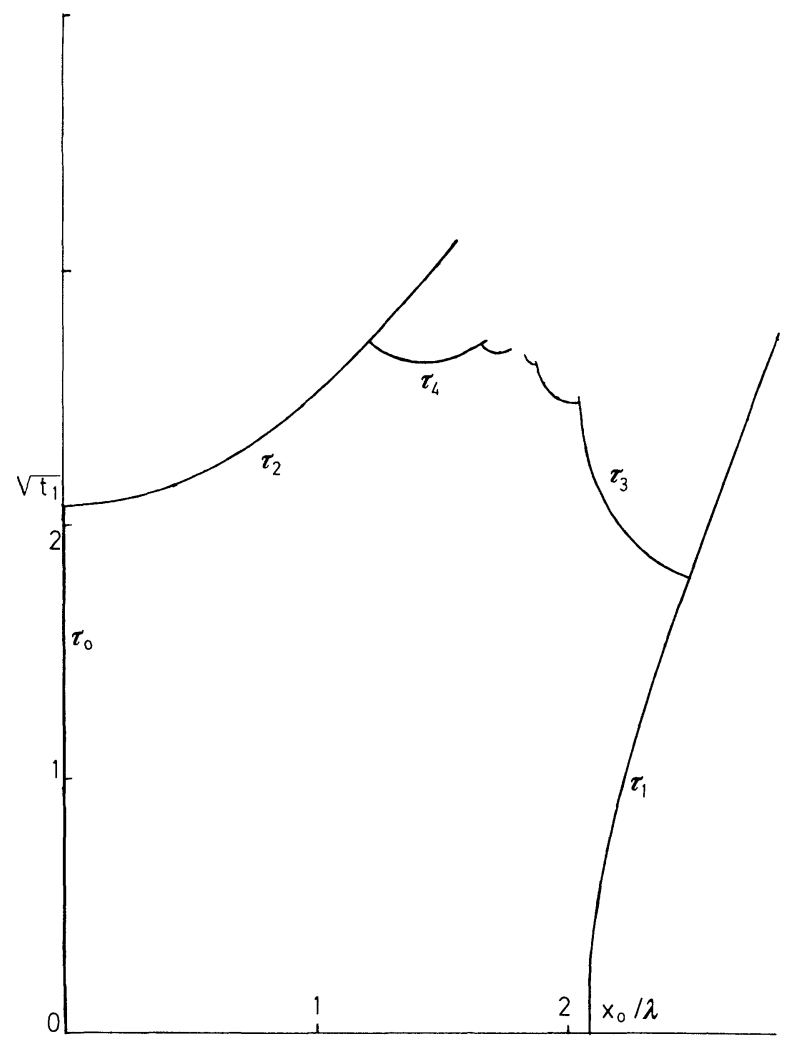

Fig. 4. Border of $u\left(\Pi_{-}\right)$

This is a Herglotz function. Starting with $n=2$, the starting point of $\tau_{n}$ is inside $\Pi_{+}$: it coincides with the end point of $\tau_{n-2} ; \tau_{n}$ and $\tau_{n-2}$ are at right angles. This is due to the fact that, at all real points in a neighborhood of $(-\lambda)^{-n}, u$ is continuous, and it has a square-root branch point at $(-\lambda)^{-n}$. Indeed if this is true for $n \leqq m-1$, it remains true for $n=m$ because, in the formula $\frac{1}{\lambda} u(u(-\lambda \zeta)), u(-\lambda \zeta)$ is inside the domain of analyticity of $\frac{1}{\lambda} u$. For the same reasons $u^{\prime}(\zeta \pm i 0) \neq 0$ whenever $\zeta \in \mathbb{R}$ and $\zeta \neq(-\lambda)^{-n}$ for all $n \geqq 0$. Note that $v_{1}$ coincides with $u_{2}$. The other $v_{n}$ are defined as self-conjugate [i.e. $\left.v_{n}(\zeta)=\bar{v}_{n}(\bar{\zeta})\right]$. However the closure of $v_{n}\left(\Pi_{+}\right)$does not intersect the real axis.

Figure 4 depicts the first few $\tau_{n}$ and exhibits the fact that $u\left(\Pi_{-}\right)$is bounded. The next subsection is devoted to a proof of this fact.

\subsection{Boundedness of $u\left(\Pi_{-}\right)$}

Consider the two maps of $\Pi_{+}$into itself given by

$$
\chi(\zeta)=\frac{1}{\lambda} u(\bar{\zeta})=\frac{1}{\lambda} \bar{u}(\zeta)
$$


and

$$
\Phi(\zeta)=\frac{1}{\lambda} u\left(\frac{1}{\lambda} u(\zeta)\right)=\chi(\chi(\zeta))
$$

The first is anti-analytic, the second analytic, both are injective and extend to continuous injective maps of $\Pi_{+}^{c}$ into itself. We have $\Phi\left(\Pi_{+}\right) \subset \frac{1}{\lambda} u\left(\Pi_{-}\right)$. If $\zeta=u(w)$,
$w \in \Pi_{-}$, then

$$
\Phi(\zeta)=\frac{1}{\lambda} u\left(\frac{1}{\lambda} u(u(w))\right)=\frac{1}{\lambda} u\left(u\left(-\frac{w}{\lambda}\right)\right)=u\left(\frac{w}{\lambda^{2}}\right)
$$

Hence $\Phi(u(w)) \in u\left(\Pi_{-}\right)$and

$$
u^{-1}(\Phi(u(w)))=\frac{w}{\lambda^{2}} \quad \text { for all } \quad w \in \Pi_{-} .
$$

This is equivalent to

$$
u \circ \lambda^{-2} \circ u^{-1}\left|u\left(\Pi_{-}\right)=\Phi\right| u\left(\Pi_{-}\right) .
$$

$u^{-1}(=g)$ is continuous on $u\left(\Pi_{-}^{c}\right)$. Hence formulae (14) and (15) remain true on $\Pi_{-}^{c}$ and on $u\left(\Pi_{-}^{c}\right)$ respectively. In particular $\Phi \mid u\left(\Pi_{-}^{c}\right)$ has an unstable fixed point at $u(0)=x_{0}$, from which emerge the invariant lines $\left\{\zeta: \zeta=u\left(\varrho e^{i \theta}\right), \varrho>0\right\},-\pi<\theta<0$. Any $\zeta \in u\left(\Pi_{-}^{c}\right)$, distinct from $x_{0}$, moves further and further away from $x_{0}$ under repeated application of $\Phi$.

We shall apply to $\Phi$ the theory of iterated Herglotz functions, due to Wolff, Denjoy, Valiron, and beautifully expounded by Valiron in [12]. We need the following facts:

a. Let $\varphi$ be a holomorphic map of $\Pi_{+}$into itself. It can be uniquely written as

$$
\varphi(\zeta)=\kappa \zeta+\psi(\zeta)
$$

$\kappa \geqq 0, \psi\left(\Pi_{+}\right) \subset \Pi_{+}$and, in any angle $\{\operatorname{Im} \zeta>k|\zeta|\}, k>0,|\psi(\zeta)| /|\zeta| \rightarrow 0$ as $\zeta \rightarrow \infty$. The constant $\kappa=\inf [\operatorname{Im} \varphi(\zeta) / \operatorname{Im} \zeta]$ is called the "angular derivative of $\varphi$ at $\infty$ ".

b. Let $\varphi_{n}$ denote the $n^{\text {th }}$ iterate of $\varphi$. There are 4 possible cases:

(i) $\varphi$ is a homographic transformation mapping $\Pi_{+}$bijectively onto itself.

In the 3 other possible cases, $\varphi_{n}$ converges, uniformly on each compact subset of $\Pi_{+}$to:

(ii) a constant $a \in \Pi_{+}$which is then an attractive fixed point of $\varphi$;

(iii) a constant $a \in \partial \Pi_{+},|a|<\infty$;

(iv) infinity. This is only possible if $\kappa \geqq 1, \kappa$ being the angular derivative of $\varphi$ at $\infty$.

In the case of $\varphi=\Phi$, manifestly not a homographic transformation of the abovementioned type, the "angular derivative at $\infty$ " is zero. Indeed $\Phi$ is the square root of a Herglotz function and cannot grow faster at infinity, in non-real directions, than $|\zeta|^{1 / 2}$. Thus cases (i) and (iv) are excluded, and therefore $\Phi_{n}$ converges, uniformly on any compact in $\Pi_{+}$, to a finite constant denoted $c$, with $\operatorname{Im} c \geqq 0$, which (since $\Phi$ is continuous in $\Pi_{+}^{c}$ ) must satisfy $\Phi(c)=c$. [In case $c$ were real this should be interpreted as $c=\Phi(c+i 0)$.] Since $u\left(\Pi_{-}\right)$is sent into itself by every $\Phi_{n}, c \in u\left(\Pi_{-}\right)^{c}$. More precisely

$$
c=\lim _{n \rightarrow \infty} \Phi_{n}\left(u\left(\varrho e^{i \theta}\right)\right)=\lim _{n \rightarrow \infty} u\left(\lambda^{-2 n} \varrho e^{i \theta}\right)
$$


the limit being uniform for $\varrho \in\left[\varrho_{1}, \varrho_{2}\right], \theta \in\left[\theta_{1}, \theta_{2}\right]$, provided $0<\varrho_{1} \leqq \varrho_{2}<\infty,-\pi<\theta_{1}$ $\leqq \theta_{2}<0$. This means that

$$
c=\lim _{\varrho \rightarrow+\infty} u\left(\varrho e^{i \theta}\right)
$$

uniformly for $\theta \in\left[\theta_{1}, \theta_{2}\right]$. This implies

$$
c=\lim _{\varrho \rightarrow+\infty} \lambda^{-1} u\left(u\left(-\lambda \varrho e^{i \theta}\right)\right) .
$$

But $u\left(-\lambda \varrho e^{i \theta}\right) \rightarrow \bar{c}$, a point of continuity of $u$. Hence

$$
c=\frac{1}{\lambda} u(\bar{c})
$$

In case $c$ is real [i.e. case (iii) occurs],(17) must be interpreted as : $\lambda c=u(c-i 0)$. But this would mean that $u(c-i 0)$ is real, i.e. $c \in\left[-\lambda^{-1}, 1\right]$, and $g(\lambda c)=c$, whence $g(c)=0$ and $c=x_{0}$. This is impossible because $x_{0} \in u\left(\Pi_{-}^{c}\right)$, where $\Phi_{n}$ is conjugated to $\lambda^{-2 n}$. Hence there is a neighborhood $\mathscr{N}$ of $x_{0}$, e.g. $\mathscr{N}=u\left(\left\{\zeta:|\zeta|<\lambda^{2}\right\}\right)$ such that, for each $\zeta \in \mathscr{N} \cap \Pi_{+}$, there exists $N>0$ such that

$$
n \geqq N \Rightarrow \Phi_{n}(\zeta) \notin \mathscr{N} \text {. }
$$

Thus $\Phi_{n}(\zeta)$ cannot tend to $x_{0}$, and case (iii) is excluded. Only case (ii) remains, so $\operatorname{Im} c>0$ and $c$ is an attractive fixed point of $\Phi$, and also an attractive periodic point of $\zeta \rightarrow \frac{1}{\lambda} u(\zeta)$. Indeed $\lambda^{-1} u(c)=\bar{c}, \quad \lambda^{-1} u(\bar{c})=c$, and $\Phi^{\prime}(\mathrm{c})=\left|\lambda^{-1} \mathrm{u}^{\prime}(\mathrm{c})\right|^{2}<1$. Hence $\left|\lambda^{-1} u^{\prime}(c)\right|<1$ and $c$ is also an attractive fixed point of $\chi$.

The open set $u_{1}\left(\Pi_{-}\right)$is a domain of analyticity for $g$. At any of the segments $\tau_{n}$ of the boundary of this domain, $g$ is continuous, takes real values, and can be continued into $v_{n}\left(\Pi_{+}\right)$. Since $\tau_{n}=\lambda^{-1} u\left(\bar{\tau}_{n-1}\right)$, these arcs converge to $c$ as $n \rightarrow \infty$. The point $c$ is a singular point of $g$ : as $\varrho \rightarrow \infty, u\left(\varrho e^{i \theta}\right) \rightarrow c,(-\pi<\theta<0)$, and $g\left(u\left(\varrho e^{i \theta}\right)\right)=\varrho e^{i \theta} \rightarrow \infty$. Each of the patches $v_{n}\left(\Pi_{+}\right)$has its version of $c$ namely

$$
\lim _{\substack{\zeta \rightarrow \infty \\ \zeta \in \Pi_{+}}} v_{n}(\zeta)=\left(\lambda^{-1} u\right)^{n}\left(-u\left((-\lambda)^{n} \zeta\right)\right)=\left(\lambda^{-1} u\right)^{n}(-c)
$$

if $n$ is odd or $\left(\lambda^{-1} u\right)^{n}(-\bar{c})$ if $n$ is even. These singular points converge to $c$. Each of them is the limit of a sequence of singular points, each of which ... etc.

The boundary of $v_{1}\left(\Pi_{+}\right)$is given by

$$
\left[\frac{x_{0}}{\lambda}, x_{1}\right] \cup\left[\bigcup_{n=0}^{\infty} \frac{1}{\lambda} u\left(-\tau_{n}\right)\right] \cup\left\{v_{1}(i \infty)\right\}
$$

(with $\lambda^{-1} x_{0}<x_{1}<\lambda^{-2} x_{0}$ ) and so

$$
\partial v_{2}\left(\Pi_{+}\right)=i\left[\sqrt{t_{1}}, y_{2}\right] \cup\left[\bigcup_{n=0}^{\infty} \frac{1}{\lambda} u\left(\frac{1}{\lambda} u\left(-\bar{\tau}_{n}\right)\right)\right] \cup\left\{v_{2}(i \infty)\right\}
$$

is contained in $\Pi_{+}$. Thus $v_{2}\left(\Pi_{+}\right)^{c}$ is a compact subset of $\Pi_{+}$, and therefore

$$
\chi^{r}\left(v_{2}\left(\Pi_{+}\right)^{c}\right)=v_{2+r}\left(\Pi_{+}\right)^{c}
$$

converges to $c$ as $r \rightarrow \infty$. 
Another consequence of the boundedness of $u$ and $U$ is that

$$
\begin{aligned}
u(\zeta) & =\frac{-1}{\pi} \int \sigma(t)\left[\frac{1}{t-\zeta}-\frac{1}{t-1}\right] d t, \\
U(\zeta) & =-\frac{1}{\pi} \int \varrho(t)\left[\frac{1}{t-\zeta}-\frac{1}{t-1}\right] d t,
\end{aligned}
$$

where $\sigma$ and $\varrho$ are positive bounded continuous functions on $\mathbb{R}$,

$$
\sigma(t)=-\operatorname{Im} u(t+i 0), \quad \varrho(t)=-\operatorname{Im} U(t+i 0),
$$

with supports

$$
\operatorname{supp} \sigma=\left[-\infty,-\lambda^{-1}\right] \cup[1, \infty], \quad \operatorname{supp} \varrho=\left[-\infty,-\lambda^{-1}\right] \cup\left[\lambda^{-2}, \infty\right],
$$

and $\sigma(t)|t-1|^{-1 / 2}$ is also continuous. For $-\lambda^{-1}<\zeta<\lambda^{-2}, n \geqq 1$,

$$
\frac{1}{n !}\left(\frac{d}{d \zeta}\right)^{n} U(\zeta)=-\frac{1}{\pi} \int \varrho(t)(t-\zeta)^{-n-1} d t
$$

This is negative for odd $n$, and furthermore for any finite sequence $\left\{a_{n}\right\}, a_{n} \in \mathbb{C}$, $n=0,1, \ldots$,

$$
-\sum_{n, m=0}^{\infty} a_{n} \bar{a}_{m}[(n+m+1) !]^{-1} U^{(n+m+1)}(\zeta) \geqq 0 .
$$

In particular, from

$$
\frac{1}{6} U^{\prime}(\zeta) U^{\prime \prime \prime}(\zeta)-\frac{1}{4} U^{\prime \prime}(\zeta)^{2} \geqq 0
$$

one recovers $S f \leqq 0$.

\subsection{Other Branches}

For $n=1,2, \ldots$ and $\varepsilon=\left(\varepsilon_{1}, \ldots, \varepsilon_{2^{n}}\right)$, with $\varepsilon_{j}= \pm 1$, let

$$
u_{\varepsilon}(\zeta)=\left(\frac{1}{\lambda}\right)^{n} \varepsilon_{1} u\left(\varepsilon_{2} u\left(\ldots \varepsilon_{2^{n}} u\left((-\lambda)^{n} \zeta\right) \ldots\right)\right)
$$

i.e.

$$
u_{\varepsilon}=\left(\frac{1}{\lambda}\right)^{n} \varepsilon_{1} u \circ \varepsilon_{2} u \circ \ldots \circ \varepsilon_{2^{n}} u \circ(-\lambda)^{n}
$$

Denote $|\varepsilon|=n$. By inserting $u=\frac{1}{\lambda} u \circ u \circ(-\lambda) 2^{n}$ times in formula (18) we re-express $u_{\varepsilon}$ as $u_{\varepsilon^{\prime}}$, with $\left|\varepsilon^{\prime}\right|=|\varepsilon|+1$ and, by induction, as $u_{\varepsilon^{\prime \prime}}$ with $\varepsilon^{\prime \prime}=|\varepsilon|+N$ for any integer $N \geqq 1$. Thus the representation (18) is far from unique. The function $u_{\varepsilon}$ is defined in $\Pi_{+} \cup \Pi_{-}$, where it is injective, self-conjugate, and Herglotz (respectively anti-Herglotz) if $(-1)^{n} \varepsilon_{1} \ldots \varepsilon_{2^{n}}=1$ (respectively -1 ). Suppose that $|\varepsilon|=n,\left|\varepsilon^{\prime}\right|=m$. If $n \leqq m$, we reexpress $u_{\varepsilon}$ as $u_{\varepsilon^{\prime \prime}}$ with $\left|\varepsilon^{\prime \prime}\right|=m$. Then

$$
\begin{aligned}
\frac{1}{\lambda} u_{\varepsilon} \circ u_{\varepsilon^{\prime}} \circ(-\lambda) & =\frac{1}{\lambda} u_{\varepsilon^{\prime \prime}} \circ u_{\varepsilon^{\prime}} \circ(-\lambda) \\
& =\left(\frac{1}{\lambda}\right)^{m+1} \varepsilon_{1}^{\prime \prime} u \circ \ldots \varepsilon_{2^{m}}^{\prime \prime} u \circ(-\lambda)^{m} \circ\left(\frac{1}{\lambda}\right)^{m} \varepsilon_{1}^{\prime} \circ \mathcal{u} \ldots \circ \varepsilon_{2^{m}}^{\prime} u \circ(-\lambda)^{m+1}
\end{aligned}
$$


is of the form $u_{\varepsilon^{\prime \prime \prime}}$ with $\left|\varepsilon^{\prime \prime \prime}\right|=m+1$. Similarly if $m<n$, we re-express $u_{\varepsilon^{\prime}}$ as $u_{\varepsilon^{\prime \prime}}$ with $\left|\varepsilon^{\prime \prime}\right|=n$, and, in all cases $\lambda^{-1} \mathcal{u}_{\varepsilon} \circ \mathcal{u}_{\varepsilon^{\prime}} \circ(-\lambda)=u_{\varepsilon^{\prime \prime \prime}}$ with $\left|\varepsilon^{\prime \prime \prime}\right|=\max (m+1, n+1)$. Let $\mathscr{G}_{n}$ be the set of such functions which can be obtained with $|\varepsilon| \leqq n$; by convention we set $\mathscr{G}_{0}=\{u,-u\}$. Note that for $|\varepsilon|>1, u_{\varepsilon}$ can always be written as $\lambda^{-1} u_{\varepsilon^{\prime}} \circ \mathcal{U}_{\varepsilon^{\prime \prime}} \circ(-\lambda)$, with $\left|\varepsilon^{\prime}\right|=\left|\varepsilon^{\prime \prime}\right|=|\varepsilon|-1$.

We now prove

Lemma 3. Assume that, for some $\zeta, \zeta^{\prime} \in \Pi_{+} \cup \Pi_{-}, u_{\varepsilon}(\zeta)=u_{\varepsilon^{\prime}}\left(\zeta^{\prime}\right)$. Then $u_{\varepsilon}=u_{\varepsilon^{\prime}}$ and $\zeta=\zeta^{\prime}$.

We may assume $|\varepsilon|=\left|\varepsilon^{\prime}\right|$. If $|\varepsilon|=0$, the hypothesis either means $u(\zeta)=u\left(\zeta^{\prime}\right)$, hence $\zeta=\zeta^{\prime}$ since $u$ is injective in $\Pi_{+} \cup \Pi_{-}$, or $u(\zeta)=-u\left(\zeta^{\prime}\right)$, which is impossible since

$$
u\left(\Pi_{+} \cup \Pi_{-}\right) \subset\{w \in \mathbb{C}: \operatorname{Re} w>0\} .
$$

Suppose now $n \geqq 1$ and

$$
\varepsilon_{1} u\left(\varepsilon_{2} u\left(\ldots \varepsilon_{2^{n}} u\left((-\lambda)^{n} \zeta\right) \ldots\right)\right)=\varepsilon_{1}^{\prime} u\left(\varepsilon_{2}^{\prime} u\left(\ldots \varepsilon_{2^{n}}^{\prime} u\left((-\lambda)^{n} \zeta^{\prime}\right) \ldots\right)\right) .
$$

Applying repeatedly the previous argument we find $\varepsilon=\varepsilon^{\prime}$ and $\zeta=\zeta^{\prime}$.

This means that the patches $u_{\varepsilon}\left(\Pi_{ \pm}\right), u_{\varepsilon^{\prime}}\left(\Pi_{ \pm}\right)$never overlap if $u_{\varepsilon}$ and $u_{\varepsilon^{\prime}}$ are truly distinct.

Lemma 4. (i) For each $\varepsilon, u_{\varepsilon}$ has continuous boundary values on both sides of the real axis.

(ii) For each $\varepsilon$, there is a locally finite set of branch points on $\mathbb{R}$, separated by open intervals $I_{\varepsilon, j}$ such that $u_{\varepsilon}$ can be analytically continued across each $I_{\varepsilon, j}$, from $\Pi_{+}$into $\Pi_{-}$and vice-versa, the continuation being again some $u_{\eta}$ (depending on $j$ ). For all $\zeta$ in any $I_{\varepsilon, j}, u_{\varepsilon}^{\prime}(\zeta \pm i 0) \neq 0$. At the branch points, $\left|u_{\varepsilon}^{\prime}\right|$ tends to $\infty$.

(iii) All $u_{\varepsilon}$ can be obtained by successive such continuations, starting from $u$.

Proof of (i) and (ii) (by induction on $|\varepsilon|$ ). Suppose (i) and (ii) hold whenever $|\varepsilon| \leqq n-1$, $n \geqq 1$. Then, for $|\varepsilon|=n, u_{\varepsilon}=\lambda^{-1} u_{\varepsilon^{\prime}} u_{\varepsilon^{\prime \prime}}$ o $(-\lambda)$, with $\left|\varepsilon^{\prime}\right|=\left|\varepsilon^{\prime \prime}\right|=n-1$, and $u_{\varepsilon}$ has continuous boundary values on both sides of $\mathbb{R}$. Let $\zeta \in \mathbb{R}:$ it is a point of analyticity of $u_{\varepsilon} \mid \Pi_{+}$unless $-\lambda \zeta$ is a branch point of $u_{\varepsilon^{\prime \prime}}$ or $u_{\varepsilon^{\prime \prime}}(-\lambda \zeta-i 0)$ is a branch point of $u_{\varepsilon^{\prime}}$. If either of these cases occurs, $\left|u_{\varepsilon}^{\prime}(w)\right|$ tends to $\infty$ as $w \rightarrow \zeta, w \in \Pi_{+}$, by the chain rule: if $-\lambda \zeta$ is a regular point of $u_{\varepsilon^{\prime \prime}}$, then $u_{\varepsilon^{\prime \prime}}^{\prime}(-\lambda \zeta-i 0) \neq 0$; if $u_{\varepsilon^{\prime \prime}}(-\lambda \zeta-i 0)$ is a regular point of $u_{\varepsilon^{\prime}}$, then $u_{\varepsilon^{\prime}}^{\prime} \neq 0$ there; if neither, then both $u_{\varepsilon^{\prime}}^{\prime}$ and $u_{\varepsilon^{\prime \prime}}^{\prime}$ tend to $\infty$. Let $I \in \mathbb{R}$ be an interval of regularity of $u_{\varepsilon} \mid \Pi_{+}$. Then, for all $\zeta \in I,-\lambda \zeta$ is a regular point for $u_{\varepsilon^{\prime \prime}} \mid \Pi_{-}$, and $u_{\varepsilon^{\prime \prime}}(-\lambda \zeta-i 0)$ is a regular point for $u_{\varepsilon^{\prime}}$, and $u_{\varepsilon}^{\prime}(\zeta+i 0) \neq 0$ by the chain rule. If $u_{\varepsilon^{\prime \prime}}\left(-\lambda \zeta_{0}-i 0\right)$ is real for some $\zeta_{0} \in I$ then $u_{\varepsilon^{\prime \prime}}(-\lambda J-i 0) \subset \mathbb{R}$ for some open real interval $J \supset \zeta_{0}$, otherwise, since $u_{\varepsilon^{\prime \prime}}^{\prime}\left(-\lambda \zeta_{0}-i 0\right) \neq 0, u_{\varepsilon^{\prime \prime}}$ cannot map $\Pi_{-}$into $\pm \Pi_{-}$, and hence $u_{\varepsilon^{\prime \prime}}(-\lambda I-i 0) \subset \mathbb{R}$. Then by Schwarz's reflection principle, $u_{\varepsilon^{\prime \prime}}$ is its own continuation across $-\lambda I$; by the induction hypothesis $u_{\varepsilon^{\prime}}$ gets continued by some $u_{\varepsilon^{\prime \prime}}$, and hence, by the composition rule, $u_{\varepsilon}$ gets continued by some $u_{\eta}$ across $I$. If $u_{\varepsilon^{\prime \prime}}(-\lambda I) \subset \Pi_{ \pm}, u_{\varepsilon^{\prime}}$ is analytic there, $u_{\varepsilon^{\prime \prime}}$ gets continued across $-\lambda I$ by some $u_{\varepsilon^{\prime \prime}}$, and, again by the composition rule, $u_{\varepsilon}$ gets continued by some $u_{\eta}$ across $I$.

Proof of (iii). Assume that, for all $\varepsilon$ with $|\varepsilon| \leqq n-1, u_{\varepsilon}$ can be obtained by analytic continuation from $u$ along a path $\gamma_{\varepsilon}$ of the following type: a finite succession of segments along the upper or lower side of the real axis, linked by judicious crossings, and such that the image of $\gamma_{\varepsilon}$ under the continuation of $u$ lies wholly in $\Pi_{+}$or $\Pi_{-}$. This 
is obviously true for $|\varepsilon| \leqq 1$, (since $\mathscr{G}_{1}=\left\{u_{1},-u_{1}, u_{2},-u_{2}=u_{-2}\right\}$ ) so we assume $n \geqq 2$. Given $\varepsilon$ with $|\varepsilon| \leqq n-1$ and any $\varepsilon^{\prime}$, the path $\gamma_{\varepsilon}$ can be used to continue $\lambda^{-1} u_{\varepsilon^{\prime}} \circ u \circ(-\lambda)$ to $\lambda^{-1} u_{\varepsilon^{\prime}} \circ u_{\varepsilon} \circ(-\lambda)$ : it suffices to let $-\lambda \zeta$ follow $\gamma_{\varepsilon}$. We now show how to obtain $\lambda^{-1} u_{\varepsilon} \circ u^{\circ}(-\lambda)$ from $\lambda^{-1} u \circ u \circ(-\lambda)=u$. For this purpose (in view of the induction hypothesis, and since $|\varepsilon| \leqq n-1)$, it will suffice to prove the following: let $u_{\eta}$ and $u_{\eta^{\prime}}$ be continuations of each other across a certain real open segment $I$, e.g. from $\Pi_{+}$into $\Pi_{-}$; then $\lambda^{-1} u_{\eta}{ }^{\circ} \mathcal{\circ} \circ(-\lambda)$ can be continued into $\lambda^{-1} u_{\eta^{\prime}} \circ \mathcal{u} \circ(-\lambda)$ in the above manner. To see this, let $\gamma_{r}$ be the already known path (see 3.1 and Fig. 3) with image in $\Pi_{+}$ permitting a continuation of $u$ into $u_{r}, r \in \mathbb{Z}$ chosen so that $u_{r}\left(\Pi_{ \pm}\right)$borders the real axis along a non-empty open subinterval of $I$, on the $\Pi_{+}$side. Let $-\lambda \zeta$ follow $\gamma_{r}$. Then $\lambda^{-1} u_{\eta} \circ u^{\circ}(-\lambda)$ gets continued into $\lambda^{-1} u_{\eta} \circ u_{r} \circ(-\lambda)$. Then let $-\lambda \zeta$ cross the real axis so that $u_{r}(-\lambda \zeta)$ crosses $I$, which it can do without leaving the domain of definition of $u_{r}$; then $\lambda^{-1} u_{\eta} \circ u_{r} \circ(-\lambda)$ gets continued by $\lambda^{-1} u_{\eta^{\prime}} \circ u_{r} \circ(-\lambda)$. Let $-\lambda \zeta$ follow the reverseconjugate path corresponding to $\gamma_{r}$. In the end we find $\lambda^{-1} u_{\eta^{\prime}} \circ u^{\circ}(-\lambda)$.

Lemma 5. The branch points of $u_{\varepsilon}$ are all of the square-root type.

Proof. In view of the definition of $u_{\varepsilon}$, the only way in which this might fail to be true is : there exist $\varepsilon_{1}, \ldots, \varepsilon_{n},\left(\varepsilon_{j}= \pm 1\right)$ and $\zeta_{0}$ such that: $\zeta_{0}$ is a branch point of $u$ and $w_{0}=\varepsilon_{1} u\left(\varepsilon_{2} u\left(\ldots \varepsilon_{n} u\left(\zeta_{0}\right) \ldots\right)\right)$ is a branch point of $u$ while, for $p=2, \ldots, n$, $\varepsilon_{p} u\left(\varepsilon_{p+1} u\left(\ldots \varepsilon_{n} u\left(\zeta_{0}\right) \ldots\right)\right)$ is a regular real point of $u$, [unless $n=1$, but we exclude this case which is easy to deal with]. The only possibility is $\varepsilon_{1}=1$ and $w_{0}=1$, with $\zeta_{0}=1$ or $-\lambda^{-1}$. But this implies $g^{n}(1)=\zeta_{0}$, hence $\zeta_{0}=1$ since $g$ maps $[-1,1]$ into itself, i.e. $g^{n+1}(0)=1$, i.e. $g^{n}(0)=0$ which is not possible for $n>1$.

\section{Final Remarks}

We call "patch" a set of the form $u_{\varepsilon}\left(\Pi_{+}\right)$or $u_{\varepsilon}\left(\Pi_{-}\right)$. Note that $u_{\varepsilon}\left(\tau_{\varepsilon} \Pi_{+}\right) \subset \Pi_{+}$, where $|\varepsilon| \geqq 1$ and $\tau_{\varepsilon}=(-1)^{n} \varepsilon_{1} \ldots \varepsilon_{2^{n}}$. Lemma 3 asserts that two patches overlap if and only if they coincide. From the proof of Lemma 4, it is clear that the boundary of $u_{\varepsilon}\left(\Pi_{+}\right)$ consists of a doubly infinite sequence of smooth arcs, each starting perpendicularly from the preceding one (because of Lemma 5). These arcs converge to $u_{\varepsilon}(+i \infty)$. Since $u_{\varepsilon}$ is injective on $\Pi_{+} \cup \Pi_{-}$, it has an inverse function, denoted $g_{\varepsilon}$ on $u_{\varepsilon}\left(\Pi_{+}\right) \cup u_{\varepsilon}\left(\Pi_{-}\right)$. The proof of Lemma 4, (iii) shows that, for any given $\varepsilon,|\varepsilon|>1$, there is a finite chain of patches, $u_{\eta(0)}\left(\Pi_{-}\right), \ldots, u_{\eta(n)}\left(\tau_{\eta(n)} \Pi_{+}\right), u_{\eta(0)}=u, u_{\eta(n)}=u_{\varepsilon}$, such that $u_{\eta(p)}\left(\tau_{\eta(p)} \Pi_{+}\right)^{c}$ and $u_{\eta(p+1)}\left(\tau_{\eta(p+1)} \Pi_{+}\right)^{c}$ have a common arc, and $u_{\eta(0)}, \ldots, u_{\eta(n)}$ are the successive continuations of $u$ along the path $\gamma_{\varepsilon^{*}}$. The various $g_{\eta(p)}$ are then successive continuations of $g$. Thus $g$ is analytic and uniform in $\bigcup_{\varepsilon} u_{\varepsilon}\left(\Pi_{ \pm}\right)$and also on all the regular arcs of the boundaries of the patches, as well as at the ends of such arcs (in view of Lemma 5), which are critical points for $g$. At points of the form $u_{\varepsilon}(i \infty), g$ is singular ( $g$ tends to infinity at such points). Note that $u_{\varepsilon}(i \infty)=\lambda^{-n} \varepsilon_{1} u\left(\ldots \varepsilon_{2^{n}} u\left((-1)^{n} i \infty\right) \ldots\right.$ ) is an image of $c$ or $\bar{c}$, hence is the limit of a sequence of patches in the same way as $c$ itself.

The preceding discussion has shown that $D=\bigcup_{\varepsilon, \pm} u_{\varepsilon}\left(\Pi_{ \pm}^{c}\right)$ is a domain of analyticity for $g$. Assume that $g$ can be continued beyond $D$, i.e. that there exists $z_{0} \in \Pi_{+}, z_{0} \notin D, \varepsilon$, $z_{1} \in u_{\varepsilon}\left(\tau_{\varepsilon} \Pi_{+}\right), R_{0}>0, R_{1}>0$, and $\hat{g}$, holomorphic in $\Omega_{0}=\left\{z:\left|z-z_{0}\right|<R_{0}\right\}$ such that

$$
\Omega_{1}=\left\{z:\left|z-z_{1}\right|<R_{1}\right\} \subset u_{\varepsilon}\left(\tau_{\varepsilon} \Pi_{+}\right)
$$




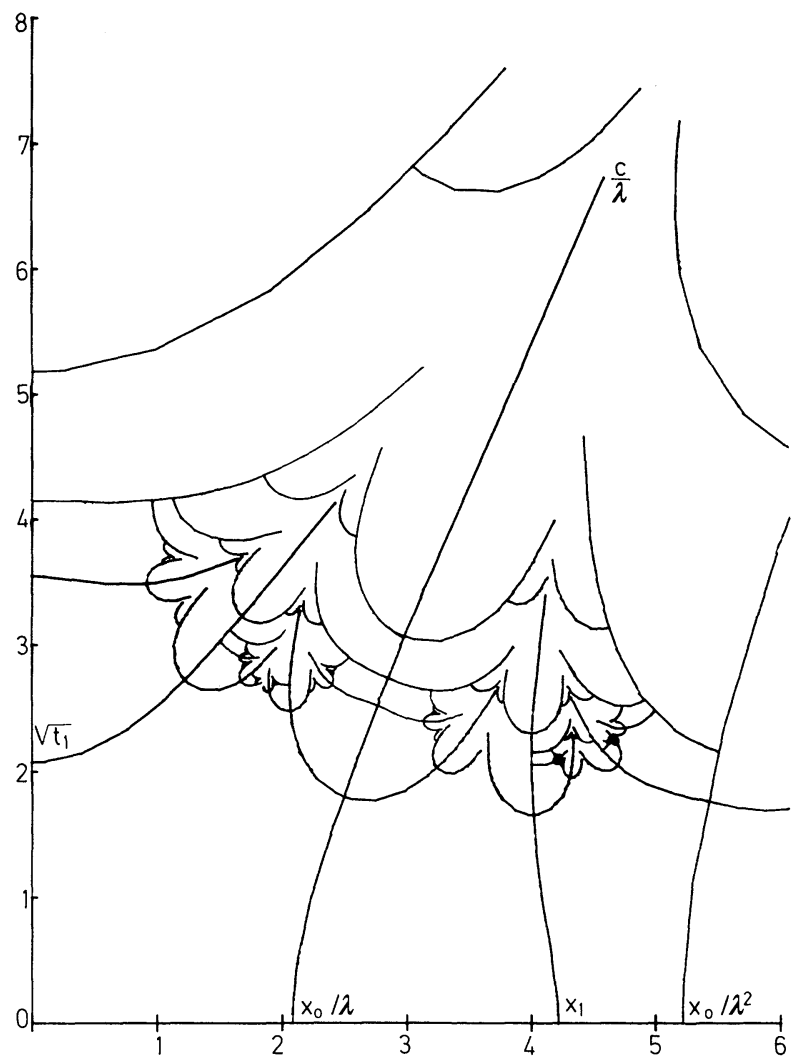

Fig. 5. Partial picture of $\{z: \operatorname{Im} g(z)=0\}$

and $\hat{g}$ coincides with $g$ in $\Omega_{0} \cap \Omega_{1} \neq \emptyset$. Then there exist $z_{1}^{\prime} \in \Omega_{0} \cap \Omega_{1}, z_{0}^{\prime} \in \Omega_{0} \backslash D$, a path $p$ from $z_{1}^{\prime}$ to $z_{0}^{\prime}$ in $\Omega_{0}$ and a neighborhood $V$ of $p$ where $\hat{g}^{\prime}$ does not vanish. One can then analytically continue $u_{\varepsilon}$ from $g\left(z_{1}^{\prime}\right)=\hat{g}\left(z_{1}^{\prime}\right)$ to $\hat{g}\left(z_{0}^{\prime}\right)$ along $\hat{g}(p)$ : therefore $z_{0}^{\prime} \in D$, a contradiction. Hence $D$ is a natural domain of holomorphy for $g$. Its boundary contains all the singular points $u_{\varepsilon}( \pm i \infty)$. If $z=u_{\varepsilon}(\zeta), \zeta \in \Pi_{+}$, then $\lambda z=u_{\varepsilon^{\prime}}\left(u_{\varepsilon^{\prime \prime}}(-\lambda \zeta)\right) \in u_{\varepsilon^{\prime}}\left(\tau_{\varepsilon^{\prime \prime}} \Pi_{-}\right)$and $g(\lambda z)=u_{\varepsilon^{\prime \prime}}(-\lambda \zeta)$. By continuity it follows that $z \in D \Rightarrow \lambda z \in D, g(\lambda z) \in D$ and $\lambda g(z)=-g(g(\lambda z))$. Figure 5 attempts to give an idea of the pattern formed by the boundary lines of the patches, i.e. the analytic set $\{z: \operatorname{Im} g(z)=0\}$. Among these lines is $\mathbb{R}$, since the patches $u_{k}\left(\Pi_{ \pm}\right), k \in \mathbb{Z}$, are bordered by the intervals $J_{k}$ (see Sect. 2). Similarly $i \mathbb{R}$ is part of the pattern : indeed, to every interval of monotonicity of $f$ on the negative real axis corresponds a restriction $f_{j}$ of $f$ which satisfies

$$
f_{j}(t)=-\frac{1}{\lambda} f_{k}\left(f_{r}\left(\lambda^{2} t\right)^{2}\right)
$$

hence a branch $U_{j}$ of $f^{-1}$ whose patches are bordered by an interval on $\mathbb{R}_{-}$; the square roots of these are some $u_{\varepsilon}$ whose patches are bordered by intervals on $i \mathbb{R}$. For any $\varepsilon$ and $\varepsilon^{\prime}, \lambda^{-1} u_{\varepsilon}{ }^{\circ} u_{\varepsilon^{\prime}} \circ(-\lambda)$ is some $u_{\varepsilon^{\prime \prime \prime}}$, hence the image of $\partial u_{\varepsilon^{\prime}}\left(\Pi_{ \pm}\right)$under $\lambda^{-1} u_{\varepsilon}$ is 
again part of the pattern; in particular, letting $u_{\varepsilon^{\prime}}$ run through the set of the $u_{k}, k \in \mathbb{Z}$ shows that the pattern is sent into itself by the dilation $\lambda^{-1}$. Similarly it contains all lines of the form $\lambda^{-1} u_{\varepsilon}(i \mathbb{R})$, which terminate at images of $c$ and $\bar{c}$. In fact, each line of the pattern other than $\mathbb{R}$ or $i \mathbb{R}$ is easily seen to be of the form $\lambda^{-N} \varepsilon_{1} u\left(\varepsilon_{2} u\left(\ldots \varepsilon_{R} u(i \mathbb{R}) \ldots\right)\right)$, and terminates at images of $\pm c$ or $\pm \bar{c}$. These line endings are points of accumulation of points of the form $u_{\varepsilon}( \pm i \infty)$. Figure 5 was obtained by using numerical data kindly provided by Lanford : the Taylor series coefficients of $U$ at 1 were obtained from his Taylor series for $f$ at 0 , then Padé-ized ; for large values of $\zeta$, the functional equation is used repeatedly ${ }^{2}$.

\section{Appendix}

We give two examples of functions $F$ on $[0,1]$ which have the properties required for the proof of Lemma 2. They are both of the form $F(x)=\lambda^{-1}[\varphi(1-x)-\varphi(1)]$, with $\varphi(x)=h\left(x^{2}\right)$ and $\varphi(0)=1, \varphi^{\prime}(1)=-\lambda^{-1}$. The verifications are straightforward. We give below (Table 2) the values of $A, c_{1}, c_{3}, \ell_{1}, \ell_{3}$, as functions of $\lambda^{2}$, and a lower bound on $L$,

$$
\begin{aligned}
& L=\left(\ell_{1}+\ell_{3} y+y^{-1}\right)\left[1-4 A^{2} y\left(1-\lambda^{4}\right)^{-1}\right]^{-1}, \\
& y=(1-A)^{2},
\end{aligned}
$$

which permit such verifications.

First Example

$$
h(t)=\frac{a}{1+\mu t}+1-a,
$$

$\mu=0.12, a$ is fixed by the condition $\varphi^{\prime}(1)=-\lambda^{-1}$ to be $a=(1+\mu)^{2} / 2 \mu \lambda$. With the above numbers $a \sim 13$.

Inverse function: the inverse function of $h$ is $V$,

$$
V(\zeta)=\mu^{-1}\left[\frac{a}{\zeta+a-1}-1\right]
$$

the inverse function of $\varphi$ is the square-root of $V$.

2 The same method leads to the following estimate for $c: c \approx 1.831259+i(2.683151)$

\section{Table 2}

\begin{tabular}{lllllll}
\hline$\lambda^{2}$ & $A$ & $c_{1}$ & $c_{3}$ & $l_{1}$ & $l_{3}$ & $L \geqq$ \\
\hline $0.152 \leqq \lambda^{2} \leqq 0.161$ & 0.26 & 0.16 & 0.224 & 0.3818 & 0.302 & 2.798 \\
$0.161 \leqq \lambda^{2} \leqq 0.164$ & 0.261 & 0.172 & 0.243 & 0.3653 & 0.3145 & 2.795 \\
$0.164 \leqq \lambda^{2}<0.165$ & 0.261 & 0.176 & 0.248 & 0.3601 & 0.3172 & 2.791 \\
\hline
\end{tabular}




\section{Second Example}

$$
h(t)=1+\frac{2 a t}{(t-b)(t-\bar{b})},
$$

$b=-r+i s, r>0, s>0, \varrho=\left[r^{2}+s^{2}\right]^{1 / 2}$ with, e.g., $r=5, \varrho=10$. The condition $\varphi^{\prime}(1)=-\lambda^{-1}$ determines a

$$
a=\left[1+2 r+\varrho^{2}\right]^{2}\left[4 \lambda\left(\varrho^{2}-1\right)\right]^{-1}
$$

( $a \sim 78$ with the above numbers).

Inverse function: the inverse function of $h$ is $V$,

$$
V(\zeta)=z-\sqrt{z^{2}-\varrho^{2}}, \quad z=-r-\frac{a}{\zeta-1},
$$

where the function $z \rightarrow \sqrt{z^{2}-\varrho^{2}}$ is defined in $\mathbb{C} \backslash[-\varrho, \varrho]$ as being asymptotic to $z$ at infinity. One also has

$$
V(\zeta)=\varrho^{2}\left[z+\sqrt{z^{2}-\varrho^{2}}\right]^{-1},
$$

which makes its anti-Herglotz character apparent.

Acknowledgement. We wish to thank Oscar Lanford for generously putting his results at our disposal in advance of publication, for help in numerical work, and for long and profitable discussions on all aspects of the subject.

\section{References}

1. Campanino, M., Epstein, H., Ruelle, D.: Topology (to appear); Campanino, M., Epstein, H.: Commun. Math. Phys. 79, 261-302 (1981)

2. Collet, P., Eckmann, J.-P.: Iterated maps on the interval as dynamical systems. Boston : Birkhäuser 1980

3. Collet, P., Eckmann, J.-P., Lanford III, O.E.: Commun. Math. Phys. 76, 211-254 (1980)

4. Coullet, P., Tresser, C.: C.R.A.S. Paris 287A, 577 (1978); J. Phys. Coll. 39, C5-25 (1978)

5. Feigenbaum, M.J.: J. Stat. Phys. 19, 25-52 (1978); 21, 669-706 (1979)

6. Feigenbaum, M.J.: Commun. Math. Phys. 77, 65-86 (1980)

7. Lanford III, O.E.: Remarks on the accumulation of period-doubling bifurcations. In : Mathematical problems in theoretical physics. Proceedings, Lausanne 1979. Berlin, Heidelberg, New York: Springer 1980

8. Lanford III, O.E.: Smooth transformations of intervals. Séminaire Bourbaki 1980/81, No. 563

9. Lanford III, O.E. : A computer-assisted proof of the Feigenbaum conjectures. IHES Preprint P/81/17 (to appear). Also: Private communication, and article in preparation

10. Lanford III, O.E.: Seminar at I.H.E.S., Bures-sur-Yvette, 1981 (to appear)

11. Rudin, W.: Real and complex analysis. New York: McGraw-Hill 1966

12. Valiron, G. : Fonctions analytiques. Paris: Presses Universitaires de France 1954 
